[56] O. KeEFe. Boston Medical and Surgeon Journal, 1920, 183, 569; 1921 , 185, 194. - Journal Amer. Med. Association, 1922, 78, 482.

[57] BRet RAtner. Journal of Immunology, 1927, 14, 275.

[58] J. BAsset. Quelques maladies infectieuses, 1946. Vigat Frères, éditeurs, Paris.

[59] E. Lemetayer, L. Nicol, O. Girard et R. Corvazier. C. R. Soc. Biol., 1948, 142, 766 .

[60] J. Courbin. Thèse de Bordeaux, 1920. Arch. de Méd. des Enfants, 1920, 23, 608.

[61] Dziengowski. Przeglad lekarski, 1903, p. 445.

[62] MCGIRr. The Veterinary Journal, 1947, 103, 345.

[63] E. Lemetayer, L. Nicol, J. Grasset, R. Gauthier et J. Pialoux. Bulletin Acad, de Méd., 1950, 134, 22.

[64] B. SALGE. Jahrbuch für Kinderheilk, 1904, 60, 175.

[65] Voir J. BAsset. Quelques maladies infectieuses, 1946. Vigot Frères, éditeurs, Paris.

[66] E. Lemetayer, L. Nicol, L. Jacob, O. Girard et R. Corvotsier. C. R. Biol. 142, 1948, 766 .

\title{
LA PASTEURISATION DU LAIT DANS LA FABRICATION DE \\ FROMAGES DE LAIT DE BREBIS ET DE CHËVRE
}

\author{
par \\ VIEIRA DE SA \\ Médecin vétérinaire. \\ Ancien boursier du Gouvernement portugais à l'Université de Reading \\ (Angleterre)
}

Je crois pouvoir soutenir que, d'une façon générale, dans la plupart des pays, il n'existe aucune obligation légale de faire de la pasteurisation du lait une opération de base pour la fabrication des fromages. Cette pasteurisation existe pourtant parfois, mais elle n'est pratiquée par les industriels que dans un but technique auquel ils sont les premiers à être intéressés; c'est done au point de vue sanitaire qu'il est nécessaire de s'en occuper.

Parmi les différents fromages que l'on fabrique habituellement, il $\mathrm{y}$ en a qui, par la nature même de leur fabrication, sont à l'abri des accusations au point de vue sanitaire. Ainsi, les fromages appelés de "pâte cuite" subissent, en raison des exigences de leur technique, une pasteurisation efficace en ce qui eoncerne les microorganismes dont la courbe de lysis se trouve au-dessous de la courbe du diagramme de North, qui indique les limites minimum de temps et de température nécessaires pour tuer Mycobacterium tuberculosis. Les "requeijoes» (fromages de lacto-albumine et de lactoglobuline) se trouvent aussi dans la même situation. De même, par la nature de la technique, mais dû à un autre procédé, on peut encore 
constater que d'autres fromages bénéficieraient de l'emploi du lait pasteurisé pour leur fabrication, ce qui permettrait d'éviter les vulgaires et presque inévitables accidents provoqués par la flore Coli-Aerogenes et motivés surtout par le peu d'acidité de la pâte, par son pourcentage d'humidité relativement élevé, et par les températures utilisées dans la fabrication. Un grand nombre de types de fromages, plus particulièrement le "Hollandais" si communément consommé en Europe, le "Saint-Paulin ou Port-Salut ", etc., se trouvent dans ce cas.

En effet, la technique industrielle a reconnu depuis longtemps que pour la réussite de son entreprise il faut autant que possible, préserver la production du fromage des fermentations anormales, d'où l'obligation de pasteuriser le lait, et en ce faisant, on pratique un acte de grande portée sanitaire.

D'autres fromages, qui dès le commencement de leur fabrication atteignent un haut degré d'acidité, ou qui sont consommés très rapidement se trouvent jusqu'à un certain point relativement préservés des maux occasionnés par les Coli-Aerogenes; pour ceux-là, les industriels relèguent à un plan secondaire, pour ne pas dire négligeable, la pasteurisation du lait. Dans ce cas se trouvent par exemple, les fromages Cheddar, Cheshire, etc., et quelques fromages à la crème fraîche qui, s'ils sont souvent fabriqués de lait pasteurisé, sont également fabriqués bien souvent sans ce traitement préalable thermique du lait. D'un autre côté, la fabrication ménagère est aussi mise en pratique, ce qui veut dire qu'elle a lieu avec du lait cru.

Or ces produits non pasteurisés constituent une continuelle menace pour la santé publique et, il n'y a pas encore longtemps, des savants canadiens ont dénoncé la gravité du mal, rattachant l'éclosion de certaines épidémies typhoïdiques au Canada à la 'consommation du fromage Cheddar fabriqué dans ce pays et par conséquent à l'emploi du lait non pasteurisé. Les savants sus-mentionnés ont voulu alors expérimenter des techniques qui permettraient d'introduire la pratique de la pasteurisation dans la fabrication du "Canadian Cheddar Chese ", sans que ses caractéristiques en fussent altérées.

Le problème ne s'impose pas particulièrement lorsque les techniques des différents types de fromage sont parfaitement connues et d'un autre côté, lorsqu'une industrialisation rationnelle est facile.

Toutefois, il me semble que le problème devient plus grave s'il s'agit du lait de brebis ou de chèvre, non seulement parce que la traite de ces animaux est faite habituellement dans des conditions hygiéniques bien pires que celles des vaches, mais encore parce que 
la fabrication des fromages de lait de brebis et de chèvres est en grande partie d'origine domestique et pastorale, ce qui signifie qu'il serait assez compliqué d'introduire la pasteurisation dans la technique de sa fabrication; cette technique étant d'ailleurs encore très peu connue et peu claire en ce qui concerne la microflore et les enzymes qui interviennent dans la maturation des produits. Même le fromage de "Roquefort" ne fait pas exception, car, quoique assez connu déjà dans les détails de sa technologie, il présente encore des altérations dans sa qualité et ses caractéristiques Iorsqu'on procède à une préalable pasteurisation du lait; voilà pourquoi on conseille - lorsqu'il y a une nécessité impérieuse de faire intervenir ce traitement - de ne le faire que dans un tiers du lait employé, les deux autres tiers demeurant sans pasteurisation afin de garantir ainsi une maturation dans de meilleures conditions.

Ces sortes de fromages de brebis, particulièrement les espagnols et les portugais pour ne pas parler de ceux de l'Europe Centrale, Italie, Grèce, etc., sont en général, fabriqués empiriquement et, par conséquent, il est techniquement impossible de conseiller leur pasteurisation si l'on ne possède pas en même temps, la connaissance des cultures et des enzymes qu'il y faut ajouter précisément.

Les produits de lait - et plus que ceux du lait de vache, ceux de brebis et de chèvre, et encore plus ceux de cette dernière espèce, par le danger de la brucellose - constituent sous le point de vue sanitaire un obstacle sérieux à la conservation ou à la préservation de la santé des populations ; j'estime donc de la plus grande importance cette opportunité d'appeler l'attention sur ce sujet, rappelant que plusieurs pays de l'Europe et d'autres continents luttent contre ce mal au dedans de leurs frontières.

Selon mon opinion, il y a deux aspects différents du problème à envisager :

10 Etudier en détail la microbiologie des susdits fromages de façon à pouvoir conseiller la pasteurisation avec innoculation ultérieure de cultures sélectionnées;

$2^{\circ}$ Etudier les possibilités économiques d'effectuer une pasteurisation à la fois efficace et pratique, dans n'importe quel cas à considérer.

Il s'agit d'une étude très poussée comprenant la chimie, la bactéorologie et la technologie laitière, afin d'atteindre les fins désirées. Il est évident que pour de telles études il faut, en outre, une connaissance complète des qualités traditionnelles des différents types de fromages; il faut aussi procéder dans les meilleures conditions de laboratoire, conditions assez coûteuses, surtout pour les pays où la production laitière ne peut justifier l'existence d'Instituts convenablement munis de matériel et de personnel spécialisés. Les 
principes à suivre dans l'investigation des meilleures techniques rationnelles sont, d'ailleurs, jusqu'à un certain point, semblables, ce qui fait qu'une somme considérable de travail intéresse un grand nombre de cas.

Il faudrait aussi défendre le point de vue que les susdites études seraient strictement réalisées dans un parfait esprit d'équipe entre les différents pays qui y seraient intéressés; les Instituts les plus renommés étant chargés de centraliser les investigations de caractère spécial et ceux qui, par leur nature, auraient besoin d'une meilleure préparation scientifique et d'un meilleur outillage.

De cette façon on serait en présence d'une grande "Commission Internationale pour l'Etude des Méthodes Rationnelles de la Fabrication de Fromages, particulièrement de lait de Brebis et de Chèvre ", dont les points essentiels d'activité seraient :

10 Connaître et classer les différents types de fromages existant dans les différents pays, notamment ceux qui sont fabriqués avec du lait de brebis et de chèvre ;

$2^{\circ}$ Etudier à fond toute la technologie des types de fromage encore peu connus, en collaboration avec les postes d'expérimentation de chaque pays;

$3^{\circ}$ Chercher à reproduire les susdits types sans interruption de caractéristiques à partir du lait pasteurisé et selon des techniques rationnelles;

4. Divulguer des techniques rationnelles.

Etant donné l'aspect artisanal ou domestique que cette industrie des fromages de lait de brebis présente le plus souvent, il convient que les solutions ne s'éloignent pas de cette réalité que le système de pasteurisation à conseiller s'accorde avec la pratique courante.

La chaleur, comme moyen d'énergie, sera-t-elle la forme la plus pratique de réaliser une pasteurisation dans de petites industries de type domestique?

Le moyen chimique sera-t-il plutôt une possibilité future pour tuer les microorganismes indésirables pour la santé sans affecter la technique de la fabrication, telle qu'on l'a fait déjà, utilisant les "catalisines" et d'autres produits pour détruire la "flore ColiAerogenes "?

Voilà ce qu'il faut rechercher afin de donner aux gouvernements la possibilité de promulguer des lois interdisant la vente des fromages de lait non pasteurisé ; en donnant, bien entendu à ce mot "pasteurisé " son sens générique, c'est-à-dire : "exempt d'organismes nuisibles à la santé ». 